\title{
Color Image Evaluation for Small Space Based on FA and GEP
}

\author{
Li Deng, ${ }^{1,2}$ Sui-Huai Yu, ${ }^{1}$ Wen-Jun Wang, ${ }^{1}$ Jun-Xuan Chen, ${ }^{1}$ and Guo-Chang Liu ${ }^{1}$ \\ ${ }^{1}$ Institute of Industrial Design, Northwestern Polytechnical University, Xi'an 710072, China \\ ${ }^{2}$ School of Mechatronic Engineering, Southwest Petroleum University, Chengdu 610500, China \\ Correspondence should be addressed to Li Deng; dengli@swpu.edu.cn
}

Received 4 November 2013; Revised 11 January 2014; Accepted 12 January 2014; Published 26 February 2014

Academic Editor: Wang Xing-Yuan

Copyright (C) 2014 Li Deng et al. This is an open access article distributed under the Creative Commons Attribution License, which permits unrestricted use, distribution, and reproduction in any medium, provided the original work is properly cited.

\begin{abstract}
Aiming at the problem that color image is difficult to quantify, this paper proposes an evaluation method of color image for small space based on factor analysis (FA) and gene expression programming (GEP) and constructs a correlation model between color image factors and comprehensive color image. The basic color samples of small space and color images are evaluated by semantic differential method (SD method), color image factors are selected via dimension reduction in FA, factor score function is established, and by combining the entropy weight method to determine each factor weights then the comprehensive color image score is calculated finally. The best fitting function between color image factors and comprehensive color image is obtained by GEP algorithm, which can predict the users' color image values. A color image evaluation system for small space is developed based on this model. The color evaluation of a control room on AC frequency conversion rig is taken as an example, verifying the effectiveness of the proposed method. It also can assist the designers in other color designs and provide a fast evaluation tool for testing users' color image.
\end{abstract}

\section{Introduction}

Rerferring to the topic of color image, color image processing has to be reviewed. It has become an important research field in computer graphics and image processing. For instance, Wang et al. proposed an effective color image retrieval method based on texture [1] and later presented a novel method for image retrieval based on structure elements' descriptor [2]. The method of image authentication [3] and restoration [4] was also been put forward. The results are plentiful and of guiding significance. However, in this paper, color image would be discussed from another perspective of Kansei engineering. Known from color ergonomics research, color affects people's emotions, feelings, thoughts, and behavior unconsciously, either direct visual stimulation or indirect association. As a kind of important visual language, different color produces different stimulus to people's physical and mental; moreover psychological effect of color affects person's mood and behavior [5]. In traditional color design, usually according to the designers' subjective experience and a lot of trials and errors to make a decision, now the color design tends to satisfy users' preferences, emphasizing feeling and experience of users. Color image is a kind of color characteristics that consider color property and color psychology together. In color design, designers must study the emotional reactions, such as the feeling associated with emotional cognitive in users' heart, and find out tacit knowledge of users [6].

As one kind of association produced by users' sense, color image relates to factors such as users' vision, experience of life, and cultural background, belongs to the category of mental activity, and can be quantitatively studied. At present, many methods that such as grey theory, fuzzy theory [7], neural network [8], genetic algorithm [9, 10], and Kansei engineering $[11,12]$ have been applied to product's form and color design. They attempted to quantify people's psychological feelings of color [13] and acquired consumers' color image finally. Hsiao and Tasi [14] used grey theory and back-propagation nerve network for children walker's color quantitative evaluation research. Hsiao et al. [15] put forward a kind of computer aided color selection system, based on the color harmony, fuzzy set theory, and so on, which could assist consumer to choose the suitable clothing color matching according to skin color and image preferences. Tsai et al. [16] proposed a kind of fast concept design method to predict 


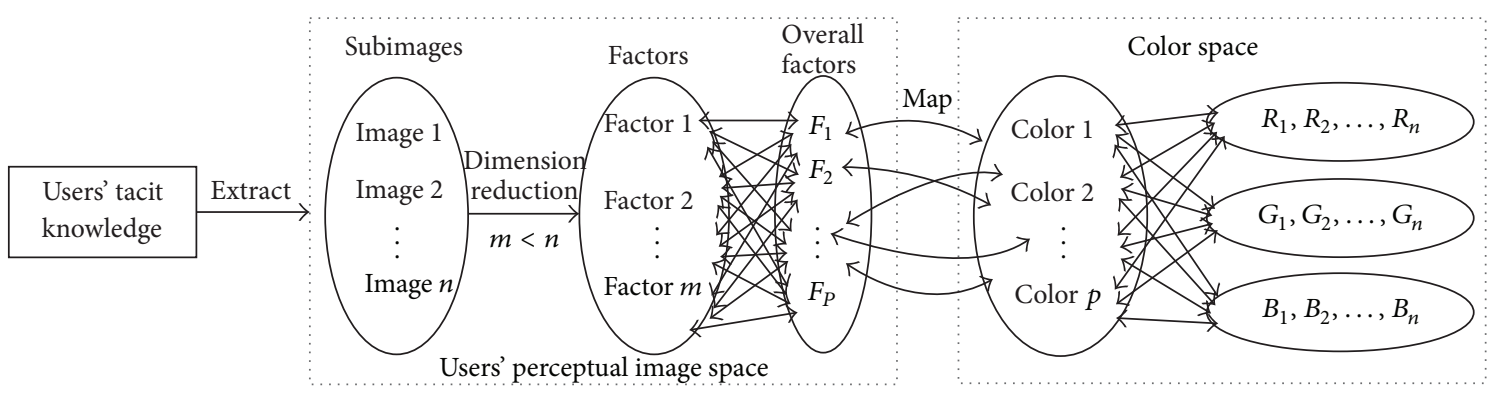

Figure 1: The mapping of users' perceptual cognitive.

image evaluation based on fuzzy neural network and grey theory and applied it in electronic lock design. Tsai and Chou [17] applied grey theory and genetic algorithm to construct an automatic design support system, which could generate color matching scheme of two colors according to the image or evaluate color scheme automatically, and it was used to evaluate the color image of kettle. Ma et al. [18] constructed a design decision-making support model for custom color combinations, which would be applied to the sofa color design.

The above research provides an efficient image evaluation criterion and auxiliary support for product color design. However, these studies mainly focus on product color design, although there are a few studies about color image and preferences of interior space [19] that hardly consider color image problem of small space. Color image is a comprehensive psychological reaction for color that is produced by a variety of sensory organs. The color of small space is different from general product appearance. Suppose a person is surrounded by environmental color, the color of small space will have strong influence on people's perception. How to validate the fact that the color image designed by designers matched with users' expectations? Users have numerous color images, and the image is fuzzy and uncertain; how to quickly and accurately reflect the nonlinear relationship between images? How to change the perceptual color design to rational calculation and then return to the understanding of perceptual once again after design? However, very few studies have discussed these issues.

Therefore, the study aims to quantitatively study the psychological attribute and emotional characteristics of color and solve the problem that users' color image perception is fuzzy. From the perspective of cognitive psychology [20], the methods such as Kansei engineering are used to make the users' implicit color preference image externalization. Aiming at the complicated nonlinear relation between the comprehensive color image and color factors, meanwhile, and considering their intrinsic relevance, excellent GEP algorithm [21] was applied to construct the relationship model between color image and color scheme, and a color optimization design method for small space was built. Finally color image evaluation system for small space was developed based on the model and assisted designers to conduct color evaluation and optimization by users' preference.

\section{Problem Description and Outline of the Method}

2.1. Problem Description. Working space [22] refers to the needed activity space when people are operating machine, plus the total space, which is occupied by machinery, equipment, appliances, tools, processing objects, and so forth. Combining with the definition of working space, the concept of small space is defined. It is a closed or semiclosed space; the range of activity for person is narrow and easily leads to oppression and other uncomfortable feelings. Such as aircraft cockpit, manned submersibles, engineering machinery cab, nuclear power plant control room, oil rig driller control room, and family car cab, in which the persons operate in sitting or standing posture. The space is restricted by various factors; besides the space of machinery and equipment layout, the activity space for person is very limited. The appropriate color design for small space will be able to adjust the space environment's atmosphere, color temperature, space size, and so on. Under the background of customization, designers need to conduct special custom to satisfy the demands of users.

Color is a sense of perception formed by the brain's visual cortex transmitted from nerve impulse, generated by light stimulate eye. The formation of users' perceptual image is a process from the divergent thinking to convergent thinking [23]. Color information seen directly and color aesthetic psychology are comprehensively processed by users; the most similar images close to the space color are searched in the memory, and users will combine several subimages to form an overall image. On the contrary, the deconstruction of the users' perceptual image is the inverse process. In order to get the users' overall image, we need to decompose and know its subimages. Because of the users' different life background, experience, culture, preferences, age, gender, and so on, perceptual images produced by different users will form a large number of subimage sets. And the formation of perceptions often accompanied by complicated mental process leads to the fact that the subjective information of images is fuzzy and uncertain. So, in order to build the mapping relationship between the perceptual information and the color design of small space, according to the users' perceptual cognitive structure of thinking (shown in Figure 1), via FA to reduce dimensionality and form the users' perceptual image space, this paper extracted users' implicit knowledge, explored the 


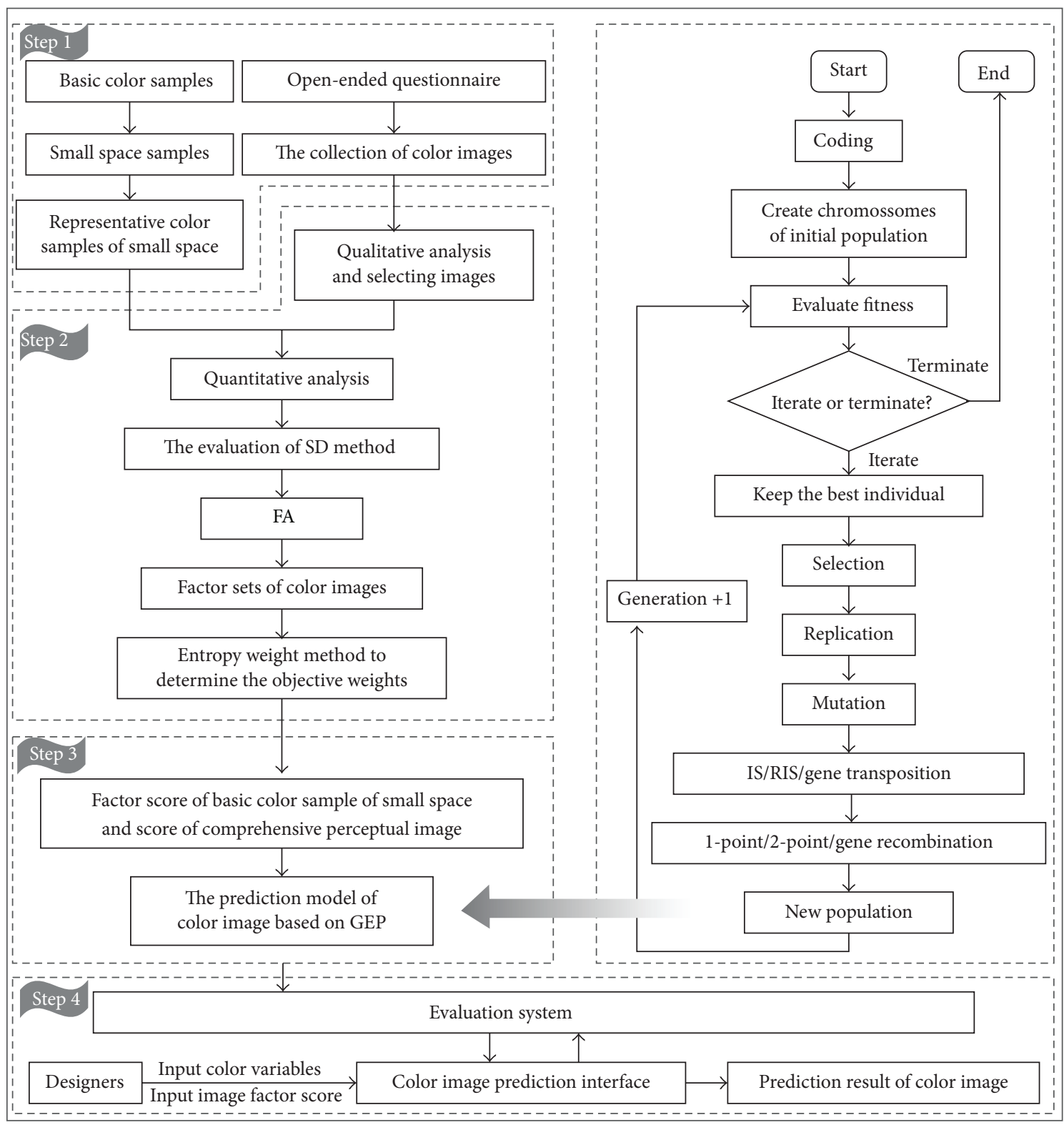

Figure 2: The color image evaluation method for small space.

relationship between the color factors and the overall image, and thus mastered users' color psychology.

2.2. Outline of the Method. As shown in Figure 2, the implementation procedures of evaluation method mainly comprise the following steps.

Step 1 (choose the basic color samples of small space and color images). The test color samples were created by regularly adjusting the RGB parameters with constant units within the range of 0-255. Human, material, and financial resources were comprehensively considered; finally, the simulation experiment method of drawing space in plane was adopted. The extensive color images were acquired by open-ended questionnaire.
Step 2 (analyze the color images qualitatively and quantitatively). The color images were qualitatively analyzed by designers and the representative adjectives were selected. Then, the perceptual image evaluation experiment was carried on by SD method [24]. Through FA to reduce dimensionality of subimage sets, factor score function was established, and the weight of each factor was determined by the entropy weight method; finally, the comprehensive perceptual image score was calculated.

Step 3 (establish the prediction model of color image based on GEP). By GEP algorithm's strong ability of function finding, the best fitting function between the color factors and comprehensive perceptual image was obtained. The function could predict the users' color image value and grasp the users' psychology. 
Step 4 (construct the color image evaluation system for small space). Based on above-mentioned model, the color image evaluation system for small space was developed, assisted designers to design the color, and provided effective tools to test users' color image.

\section{Prediction Model of Color Image for Small Space Based on FA and GEP}

\subsection{Theoretical Background}

3.1.1. FA. FA [25] is a method of multivariate statistical analysis, which studies how to make numerous original variables condense into a few factors with the least amount of information loss and how to make the factors have certain named explanatory. Suppose that there are $n$ original variables $x_{1}, x_{2}, x_{3}, \ldots, x_{n}$, after standardized treatment, variables are expressed by $m$ factors $(m<n)$, by means of linear combination of $f_{1}, f_{2}, f_{3}, \ldots, f_{m}$ :

$$
\begin{gathered}
x_{1}=a_{11} f_{1}+a_{12} f_{2}+\cdots+a_{1 m} f_{m}+\varepsilon_{1} \\
x_{2}=a_{21} f_{1}+a_{22} f_{2}+\cdots+a_{2 m} f_{m}+\varepsilon_{2} \\
\vdots \\
x_{n}=a_{n 1} f_{1}+a_{n 2} f_{2}+\cdots+a_{n m} f_{m}+\varepsilon_{n},
\end{gathered}
$$

where $a_{i j}(i=1,2, \ldots, n ; j=1,2, \ldots, m)$ represents factor loading and $\varepsilon_{i}(i=1,2, \ldots, n)$ represents special factor.

The factor loading is deduced with the method of principal component analysis (PCA). By means of coordinate transformation, after standardization, the original relevant variables $x_{1}, x_{2}, x_{3}, \ldots, x_{n}$ were linearly combined, converted into another set of uncorrelated variables $y_{i}$ :

$$
\begin{gathered}
y_{1}=\mu_{11} x_{1}+\mu_{12} x_{2}+\cdots+\mu_{1 n} x_{n} \\
y_{2}=\mu_{21} x_{1}+\mu_{22} x_{2}+\cdots+\mu_{2 n} x_{n} \\
\vdots \\
y_{n}=\mu_{n 1} x_{1}+\mu_{n 2} x_{2}+\cdots+\mu_{n n} x_{n},
\end{gathered}
$$

where $y_{1}, y_{2}, \ldots, y_{n}$, respectively, represent the first, second,..., $n$th principal component of the original variables. $y_{1}$ has the largest proportion in total variance, so it has the strongest ability to synthesize the information of original variables. The rest of principal components' ability to synthesize information declines in turn. FA can reduce the variable dimension and eliminate the correlation between the variables, so as to reduce the input of GEP independent variable in the late.

3.1.2. Entropy Weight Method. German physicist R. Clausius put forward entropy and applied it in thermodynamics. C. E. Shannon introduced the concept of entropy in information theory later, as a measurement of uncertainty or information of random events [26]. In information theory, entropy is used to measure the disorder degree of system. When the system is more orderly, the information entropy is smaller; oppositely, the information entropy is larger. The system has a variety of different state and the probability of each state to appear with the $P_{i}(i=1,2, \ldots, m)$, and then the entropy of the system is defined as

$$
e=-\sum_{i=1}^{m} P_{i} \cdot \ln P_{i}
$$

When $P_{i}=1 / m(i=1,2, \ldots, m)$, that is to say, the presence of the probability to each state is equal, the maximum entropy is $e_{\max }=\ln m$.

This paper introduced the information entropy to determine the weights in the color image evaluation for small space. If there are $m$ color samples of small space and $n$ color factors, the data matrix of original evaluation $R=\left(r_{i j}\right)_{m \times n}$ is as follows:

$$
R=\left[\begin{array}{cccc}
r_{11} & r_{12} & \cdots & r_{1 n} \\
r_{21} & r_{22} & \cdots & r_{2 n} \\
\cdots & \cdots & \cdots & \cdots \\
r_{m 1} & r_{m 2} & \cdots & r_{m 4}
\end{array}\right]_{m \times n}
$$

A color factor $r_{j}$ has information entropy $e_{j}=-\sum_{i=1}^{m} P_{i j}$. $\ln P_{i j}$ and $P_{i j}=r_{i j} / \sum_{i=1}^{m} r_{i j}(i=1,2, \ldots, m ; j=1,2, \ldots, n)$.

Calculation steps of entropy weights are as follows.

Step 1. Calculate the $j$ th color factor of the $i$ th color sample of small space's index weight $P_{i j}: P_{i j}=r_{i j} / \sum_{i=1}^{m} r_{i j}$.

Step 2. Calculate the entropy of the $j$ th color factor $e_{j}: e_{j}=$ $-k \sum_{i=1}^{m} P_{i j} \cdot \ln P_{i j}$, and $k=1 / \ln m$.

Step 3. Calculate the deviation degree of the $j$ th color factor (difference coefficient) $g_{j}: g_{j}=1-e_{j}$.

Step 4. Calculate the entropy of $j$ th factor $w_{j}: w_{j}=g_{j} /$ $\sum_{j=1}^{n} g_{j}$.

3.1.3. GEP Algorithm. GEP is a new random search and optimization algorithm, invented and put forward by the Portuguese scientist Ferreira [27]. GEP combines the advantages of genetic algorithm and genetic programming algorithm. It is a new kind of efficient and ideal data mining technique, taking the biotic genetic gene expression patterns as reference. Using adaptive random search method, and without relying on any prior knowledge, GEP can discover formula which can describe data inherent law from the data and shows strong universality and accuracy. In GEP, a computer program is coded into fixed length of linear symbol strings (chromosome), while in the individual fitness calculation, it will be expressed to different shapes and sizes of expression trees, so as to solve the problem. As shown in Figure 2, the main steps of using GEP for function mining are as follows [28].

Step 1. Code the individual and create the initial population. Population contains a number of individuals (chromosomes). 
Chromosome is comprised of more than one gene, and the genes are connected through linking function. Gene is comprised of head and tail. Head consists of function set or terminal set, and end consists of terminal set.

Step 2. Calculate the fitness. The fitness of each individual is calculated through fitness function. Fitness reflects the extent of excellence of individual to achieve the optimal solution in the course of evolution. If the optimal individual satisfies end condition, it will be transferred to the output. If not, transferred to genetic operation steps and then produces offspring with new characteristics.

Step 3. Do a series of genetic operation to produce a new generation of population, using principle of survival of the fittest to guide the evolution, including (1) keep the best individual, (2) selection, (3) replication, (4) mutation, (5) transposition, and (6) recombination.

3.2. Prediction Model of Color Image for Small Space. The core technology of GEP is that the mutation process is completely separated from the evaluation process. Fixed linear string is used in mutation process, and expression tree is used in evaluation process.

First of all, determine the symbols representing chromosome; namely, select function set and terminal set which suited the solution. GEP $=(F, T)$. Suppose function set $F=\{+,-, *, /, \sin , \cos \}$ and common function include arithmetic operators, elementary mathematics function, Boolean operator, and relational operators; terminal set $T=$ $\left\{x_{1}, x_{2}, x_{3}, \ldots, x_{n}\right\}, x_{1}, x_{2}, x_{3}, \ldots, x_{n}$ represents color image factor set.

Secondly, determine the genetic structure and the length of gene head. According to the complexity of the problem to define the length of gene head $h$, the length of tail $t$ and the length of head $h$ satisfy the relationship that $t=h(n-1)+1$. In the formula, $n$ represents the maximum number of operation numbers in function set, namely, the number involved in the operation variable. It can guarantee that the genes are legitimate in this way. Suppose that a gene is composed of the elements in $\left\{+,-, *, /\right.$, sin, $\left.\cos , x_{1}, x_{2}, x_{3}, x_{4}, x_{5}, x_{6}\right\}$, so $n=2$. If $h=6$, then $t=6(2-1)+1=7$. Randomly generate a legitimate GEP gene as follows:

$$
\sin -x_{5}^{* *}+x_{4} x_{1} x_{2} x_{3} x_{6} x_{3} x_{5} \text {. }
$$

The italicized part represents the tail. The corresponding expression tree is shown in Figure 3.

The length of each gene is fixed in GEP coding. Gene is made up of two parts: the front effective K-expression and the back of filler components. From top to bottom, left to right sequence, the expression trees in Figure 3 are traversed, and the $\mathrm{K}$-expression of expression trees is obtained. The effective length of the gene has 10 characters: $\sin -x_{5}{ }^{* *}+x_{4} x_{1} x_{2} x_{3}$. So the mathematical expression is as follows:

$$
\sin \left(x_{5}-x_{4} x_{1}\left(x_{2}+x_{3}\right)\right) \text {. }
$$

Again, determine the number of genes in each chromosome and the linking function to connect the genes.

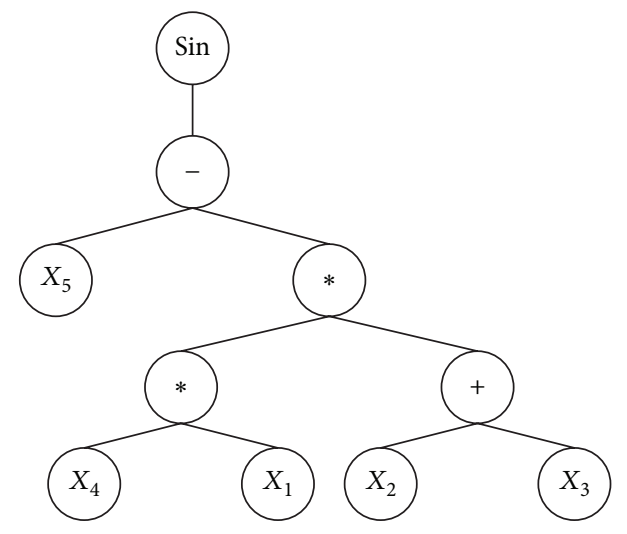

FIGURE 3: Gene expression tree.

Fourth, select the fitness function. There are three kinds of classical fitness function in GEP algorithm. According to the characteristics of the problem and reference [29], the fitness function is defined as follows:

$$
f_{i}=\frac{1000}{E_{i}+1} .
$$

In (7), $E_{i}=(1 / m) \sum_{j=1}^{m}\left(C_{i j}-T_{j}\right)^{2}$ is the mean square error (MSE) of experimental samples. $m$ is the total number of training set samples; $C_{i j}$ is the output value of the $j$ th sample of the $i$ th individual, calculated by the mathematical expressions acquired by GEP algorithm modeling; $T_{j}$ is the target value of the $j$ th sample.

Finally, determine the genetic control parameters before the algorithm running, including the size of the population, evolution generation limit, and the probability of each genetic operators.

\section{Implementation Procedures}

The effectiveness and feasibility of the proposed method are demonstrated by taking the case of color image evaluation of a control room on AC frequency conversion rig for illustration purposes. The steps involved in implementing the evaluation system are described in the following sections.

\subsection{Basic Color Samples of Small Space}

4.1.1. Basic Color Samples. The amount of basic color samples should not only reduce the workload of experiment but also not affect the measure conditions to express image. According to [17], to set color intervals, RGB parameters change with 64 units within the range of $0-255$. Each primary axis gets 5 intervals; eventually $5^{3}=125$ sets of basic color samples are obtained (shown in Figure 4), defined as $C=$ $\left\{C_{1}, C_{2}, \ldots, C_{n}\right\}$.

4.1.2. Small Space Samples. In order to avoid the mutual influence of multivariable, this paper only discusses the main color of small space and does not consider the impact of factors such as material and form for the moment. As shown in Figure 5, the space sample with transparent background 
TABLE 1: Color images obtained from questionnaire.

\begin{tabular}{|c|c|c|c|c|c|}
\hline (1) Modern & (6) Gorgeous & (11) Elegant & (16) Quiet & (21) Warm & (26) Lively \\
\hline (2) Dynamic & (7) Noble & (12) Graceful & (17) Solemn & (22) Soft & (27) Lightful \\
\hline (3) Simple & (8) Male & (13) Fresh & (18) Classical & (23) Joyful & (28) Bright \\
\hline (4) Technological & (9) Handsome & (14) Cool & (19) Plain & (24) Romantic & (29) Roomy \\
\hline (5) Fashionable & (10) Female & (15) Steady & (20) Cozy & (25) Popular & \\
\hline
\end{tabular}

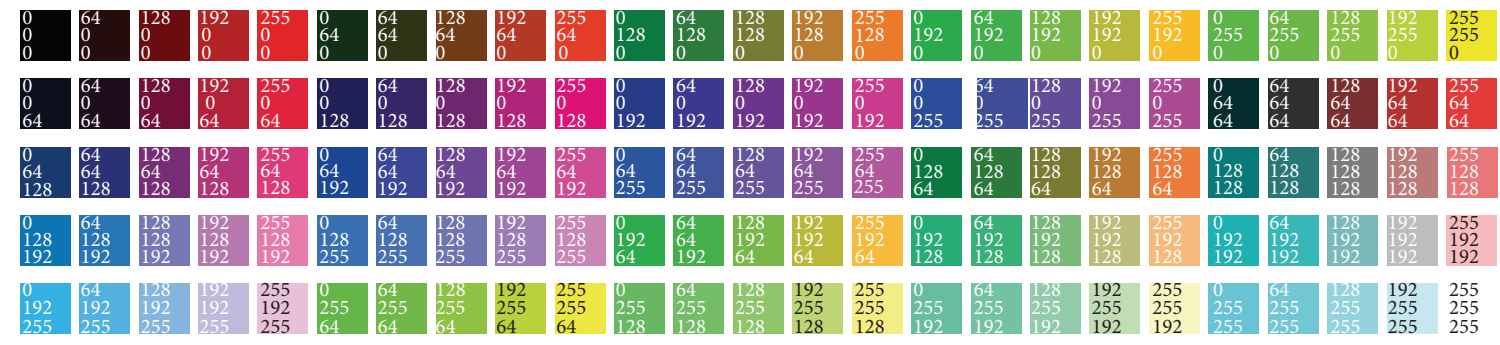

FIgURE 4: RGB values of 125 basic color samples.

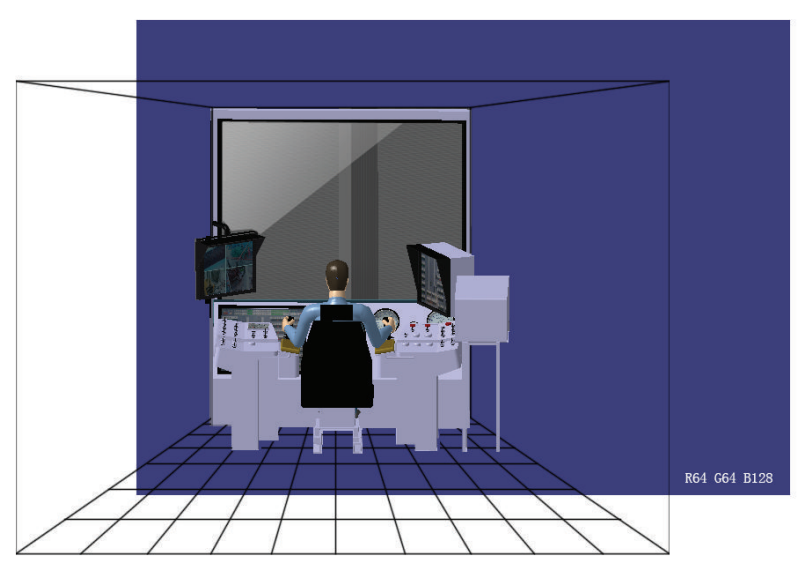

FIGURE 5: Master page of the space sample.

is made as a master page. In test process, the random superposition of the 125 color samples, which can form the color samples of small space to meet the experimental demand is shown in Figure 6.

\subsection{Qualitative and Quantitative Analysis of Color Image}

4.2.1. Qualitative Analysis. Through open-ended questionnaire [30] to acquire extensive color images, they were qualitatively analyzed by designers, according to their practical work experience. The repeated meaning words were deleted; and the similar meaning words were merged. Ultimately the selected representative adjectives covered color semantic cognitive space as much as possible. 29 color images are preliminary selected (shown in Table 1).

4.2.2. Quantitative Analysis. 40 subjects (half male and half female) are invited to take part in the experiment. All of them are students, aged between 20 and 28 years old, and both eyesight and color vision are normal. As shown in Figures 7 and 5 points psychology scale is used to represent the different dimensions of psychological variation. The evaluation value of color image for small space is set between 0 and 1 ; the larger value represents the more close to the feeling of color image.

The color samples of small space randomly are displayed on the computer screen and the subjects evaluate them according to the 29 color images, respectively. The experimental results are analyzed with SPSS Statistics19.0 software for FA. First by calculating the correlation coefficient matrix, anti-image correlation matrix, Bartlett's test of sphericity, and Kaiser-Meyer-Olkin (KMO) test, the relationship between variables is tested. The statistics observed value is 5456.468 in Bartlett's test of sphericity; since the corresponding probability of $P$ value is close to 0 , less than the significance level of $\alpha$ ( $\alpha$ equal to 0.05 ), it can be regarded as that there is significant difference between the correlation coefficient matrix and unit matrix. The value of KMO is 0.886 ; according to the KMO metrics provided by Kaiser, the original variables are suitable to conduct FA.

According to the principle [25] that "usually select the number of eigenvalues as factor number when cumulative variance contribution rate is greater than 0.85 ," the factors are extracted by the method of PCA. Six factors of color image are extracted; the corresponding cumulative variance contribution rate reaches $87.289 \%$ (shown in Table 2) and meets the above principle.

In Table 3, the data shown in bold in each column represent the color images as having high loading on the 6 factors, respectively. For example, the fifth factor mainly explains four color images: the lightful, bright, lively, and roomy. Therefore, we can summarize some conclusions that the first factor mainly explains the feeling of being modern and fashionable, the second factor mainly explains the feeling of being relaxed and calm, the third factor mainly explains the feeling of being sweet and elegant, the fourth factor mainly 
TABLE 2: Total variance explained.

\begin{tabular}{|c|c|c|c|c|c|c|c|c|c|}
\hline \multirow{2}{*}{ Component } & \multicolumn{3}{|c|}{ Initial eigenvalues } & \multicolumn{3}{|c|}{ Extraction sums of squared loadings } & \multicolumn{3}{|c|}{ Rotation sums of Squared loadings } \\
\hline & Total & $\%$ of variance & Cumulative \% & Total & $\%$ of variance & Cumulative $\%$ & Total & $\%$ of variance & Cumulative \% \\
\hline 1 & 13.085 & 45.119 & 45.119 & 13.085 & 45.119 & 45.119 & 5.757 & 19.851 & 19.851 \\
\hline 2 & 4.529 & 15.618 & 60.737 & 4.529 & 15.618 & 60.737 & 5.129 & 17.687 & 37.538 \\
\hline 3 & 3.611 & 12.450 & 73.187 & 3.611 & 12.450 & 73.187 & 4.404 & 15.185 & 52.723 \\
\hline 4 & 2.196 & 7.573 & 80.761 & 2.196 & 7.573 & 80.761 & 3.643 & 12.562 & 65.285 \\
\hline 5 & 1.036 & 3.574 & 84.335 & 1.036 & 3.574 & 84.335 & 3.347 & 11.541 & 76.826 \\
\hline 6 & 0.857 & 2.954 & 87.289 & 0.857 & 2.954 & 87.289 & 3.034 & 10.463 & 87.289 \\
\hline 7 & 0.650 & 2.242 & 89.531 & & & & & & \\
\hline 8 & 0.565 & 1.949 & 91.480 & & & & & & \\
\hline$\vdots$ & $\vdots$ & $\vdots$ & $\vdots$ & & & & & & \\
\hline 28 & 0.018 & 0.062 & 99.959 & & & & & & \\
\hline 29 & 0.012 & 0.041 & 100.000 & & & & & & \\
\hline
\end{tabular}

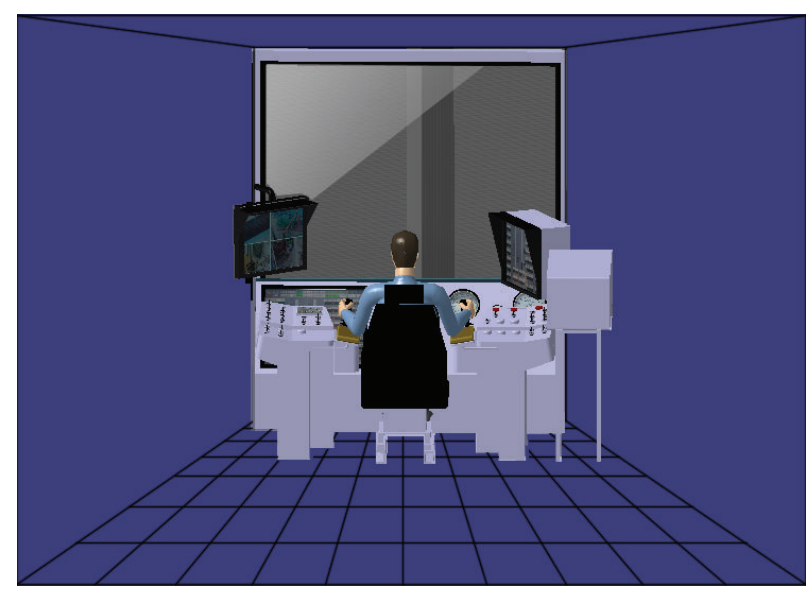

FIgURE 6: An example of the color sample of small space.

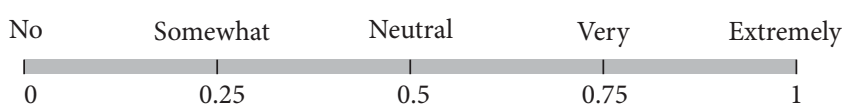

FIGURE 7: The image scale of color evaluation words.

explains the feeling of personality and being solemn, the fifth factor main explains the feeling of being bright and roomy, and the sixth factor main explains the feeling of being gorgeous and noble. In this way, the dimension of 29 variables is reduced to 6 factors and can reflect most of the information of the original variables.

Regression method is used to estimate the factor score coefficient. Component sore coefficient matrix is calculated, and the function of factor score is expressed as follows:

$$
\begin{aligned}
F_{i 1}= & 0.327 * \text { image } 1+0.104 * \text { image } 2 \\
& +\cdots-0.028 * \text { image } 28+0.037 * \text { image } 29 \\
F_{i 2}= & -0.147 * \text { image } 1-0.017 * \text { image } 2 \\
& +\cdots+0.008 * \text { image } 28+0.008 * \text { image } 29
\end{aligned}
$$

$$
\begin{aligned}
F_{i 3}= & 0.019 * \text { image } 1-0.208 * \text { image } 2 \\
& +\cdots+0.046 * \text { image } 28+0.068 * \text { image } 29 \\
F_{i 4}= & -0.112 * \text { image } 1-0.260 * \text { image } 2 \\
& +\cdots+0.083 * \text { image } 28+0.065 * \text { image } 29 \\
F_{i 5}= & -0.080 * \text { image } 1+0.162 * \text { image } 2 \\
& +\cdots+0.285 * \text { image } 28+0.184 * \text { image } 29 \\
F_{i 6}= & -0.048 * \text { image } 1+0.216 * \text { image } 2 \\
& +\cdots+0.030 * \text { image } 28-0.006 * \text { image } 29 .
\end{aligned}
$$

The factor score of each color sample of small space can be calculated by (8), then the method of calculating total score by factor weight is adopted, and the overall image is assessed comprehensively. Determining the weight of the factors is the key. Based on the principle of information entropy and the original evaluation data information, this paper used the differences between the values which reflected "information 
TABLE 3: Rotated component matrix.

\begin{tabular}{|c|c|c|c|c|c|c|}
\hline & \multicolumn{6}{|c|}{ Component } \\
\hline & 1 & 2 & 3 & 4 & 5 & 6 \\
\hline Image 1 & 0.902 & 0.180 & -0.120 & 0.210 & -0.023 & 0.034 \\
\hline Image 5 & 0.856 & 0.141 & -0.201 & 0.267 & 0.106 & 0.141 \\
\hline Image 3 & 0.814 & 0.332 & -0.209 & 0.191 & 0.177 & -0.103 \\
\hline Image 25 & 0.793 & 0.410 & 0.017 & 0.117 & 0.111 & -0.249 \\
\hline Image 4 & 0.720 & 0.435 & -0.293 & 0.349 & 0.068 & -0.051 \\
\hline Image 18 & 0.480 & 0.348 & -0.049 & 0.439 & -0.368 & 0.416 \\
\hline Image 19 & 0.458 & 0.174 & 0.054 & 0.429 & -0.361 & 0.420 \\
\hline Image 13 & 0.311 & 0.908 & 0.045 & 0.051 & 0.132 & -0.119 \\
\hline Image 14 & 0.337 & 0.864 & -0.086 & 0.166 & 0.168 & -0.207 \\
\hline Image 16 & 0.307 & 0.790 & -0.209 & 0.406 & 0.063 & -0.148 \\
\hline Image 15 & 0.400 & 0.650 & -0.191 & 0.540 & -0.099 & -0.037 \\
\hline Image 23 & 0.613 & 0.637 & 0.130 & 0.114 & 0.179 & -0.275 \\
\hline Image 20 & 0.020 & -0.027 & 0.912 & -0.115 & 0.027 & 0.150 \\
\hline Image 24 & -0.115 & 0.085 & 0.835 & -0.066 & -0.030 & 0.023 \\
\hline Image 22 & -0.117 & -0.218 & 0.803 & -0.253 & 0.116 & 0.048 \\
\hline Image 11 & -0.188 & -0.035 & 0.679 & -0.060 & -0.177 & 0.575 \\
\hline Image 21 & -0.208 & -0.541 & 0.598 & -0.358 & 0.073 & 0.208 \\
\hline Image 9 & 0.356 & 0.137 & -0.328 & 0.821 & 0.041 & -0.093 \\
\hline Image 8 & 0.440 & 0.329 & -0.351 & 0.703 & 0.047 & -0.142 \\
\hline Image 10 & -0.315 & -0.181 & 0.546 & -0.642 & -0.075 & 0.167 \\
\hline Image 17 & 0.416 & 0.561 & -0.191 & 0.609 & -0.146 & 0.031 \\
\hline Image 27 & 0.233 & 0.231 & 0.017 & 0.092 & 0.871 & -0.165 \\
\hline Image 28 & 0.239 & 0.236 & -0.001 & 0.033 & 0.853 & -0.234 \\
\hline Image 26 & -0.265 & -0.167 & 0.003 & -0.166 & 0.777 & 0.006 \\
\hline Image 29 & 0.472 & 0.403 & 0.011 & 0.187 & 0.603 & -0.220 \\
\hline Image 2 & 0.047 & -0.397 & -0.330 & -0.457 & 0.524 & 0.255 \\
\hline Image 6 & -0.043 & -0.231 & 0.120 & -0.171 & -0.001 & 0.864 \\
\hline Image 7 & -0.002 & -0.315 & 0.227 & 0.005 & -0.221 & 0.798 \\
\hline Image 12 & -0.111 & 0.007 & 0.611 & -0.070 & -0.265 & 0.631 \\
\hline
\end{tabular}

TABLE 4: The weight of factors.

\begin{tabular}{lcccccc}
\hline Weight & Factor 1 & Factor 2 & Factor 3 & Factor 4 & Factor 5 & 0.115 \\
\hline$w_{\text {si }}$ & 0.199 & 0.177 & 0.152 & 0.126 & 0.105 \\
$w_{\text {oi }}$ & 0.105 & 0.154 & 0.135 & 0.378 & 0.089 & 0.138 \\
$w_{i}$ & 0.148 & 0.193 & 0.146 & 0.338 & 0.073 \\
\hline
\end{tabular}

value" of factor to determine the weight. SD method is used to quantify the color image of subjects' preferences; evaluation data is sorted and described as follows:

$$
\left[\begin{array}{ccccc}
x_{11}^{k} & x_{12}^{k} & x_{13}^{k} & \cdots & x_{1 n}^{k} \\
x_{21}^{k} & x_{22}^{k} & x_{23}^{k} & \cdots & x_{2 n}^{k} \\
\cdots & \cdots & \cdots & x_{i j}^{k} & \cdots \\
x_{m 1}^{k} & x_{m 2}^{k} & x_{m 3}^{k} & \cdots & x_{m n}^{k}
\end{array}\right],
$$

where $m$ represents the number of color samples of small space; $n$ represents the number of factors; $x_{i j}^{k}$ represents the $k$ th subject's preference to the $j$ th factor of the $i$ th color sample of small space.

According to the above-mentioned calculation steps of entropy weights, the objective weight of each factor can be calculated, denoted as $w_{o i}=\left(w_{o 1}, w_{o 2}, \ldots, w_{o n}\right)$. Entropy weight method is a kind of objective method. In order to retain experts and policymakers' subjective opinions, namely, balance subjective and objective aspect, the entropy weight method is combined with each factor variance contribution rate in the above-mentioned FA. Suppose the variance contribution rate calculated in FA is subjective weight $w_{s i}$ and entropy $w_{o i}$ is the objective weight; the combination weight $w_{i}$ of the $i$ th factor can be calculated as follows:

$$
w_{i}=\frac{w_{s i} w_{o i}}{\sum_{i=1}^{n} w_{s i} w_{o i}} .
$$

Table 4 shows the calculation results of weights. The comprehensive color image evaluation of small space is as follows:

$$
F_{i}=w_{1} F_{i 1}+w_{2} F_{i 2}+w_{3} F_{i 3}+w_{4} F_{i 4}+w_{5} F_{i 5}+w_{6} F_{i 6} .
$$

4.3. Color Image Prediction Based on GEP. 84 groups of data were randomly selected from 125 groups of experimental data as the training set and the rest as a validation set. Table 5 shows the main operation parameters in GEP model. In function set, Sqrt represents square root; $X 2$ represents square; Avg. 2 represents the mean of two variables; 
Sub-ET 1

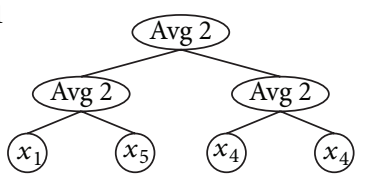

Sub-ET 2

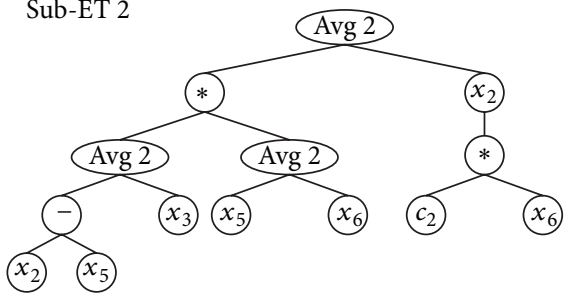

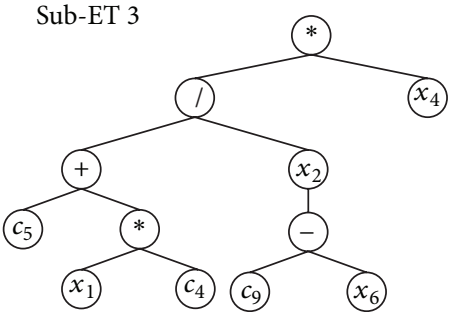

Figure 8: The sub-ET of optimal individual.
TABLE 5: GEP parameter set.

\begin{tabular}{lc}
\hline Parameter names & Parameter values \\
\hline Function set & $F=\{+,-, *, /$, Sqrt, $X 2$, Avg2, Neg, Inv $\}$ \\
Terminal set & $T=\left\{x_{1}, x_{2}, x_{3}, x_{4}, x_{5}, x_{6}\right\}$ \\
Generation & 1000 \\
Number of chromosomes & 30 \\
Number of genes & 3 \\
Linking function & + \\
Head size & 8 \\
Mutation rate & 0.002 \\
One-point/Two- & 0.003 \\
point/gene & \\
recombination rate & 0.005 \\
IS/RIS/gene & $(-10,10)$ \\
transposition rate & \\
Numerical constant &
\end{tabular}

Neg represents negative; Inv represents inverse. In terminal set, $x_{1}, x_{2}, x_{3}, x_{4}, x_{5}, x_{6}$, respectively, represent the 6 factors obtained from FA. The visual basic programming is used to express the GEP algorithm, and the program runs multiple times to get the best individual. The best individual's expression tree is shown in Figure 8. Each expression tree represents a gene, and genes are connected by linking function "+" to form a chromosome. The best individual translated into mathematical expression is as follows:

$$
\begin{aligned}
F(x)= & \frac{1}{2}\left(\frac{x_{1}+x_{5}}{2}+\frac{x_{4}+x_{4}}{2}\right) \\
& +\frac{1}{2}\left(\frac{x_{2}-x_{5}+x_{3}}{2} \cdot \frac{x_{5}+x_{6}}{2}+\left(0.3714 x_{6}\right)^{2}\right) \\
& +\frac{-4.9771-2.7711 x_{1}}{\left(-5.1091-x_{6}\right)^{2}} \cdot x_{4} .
\end{aligned}
$$

Figure 9 shows the curve fitting of GEP algorithm in the training set. MSE and $R$-square are used to verify the validity of the algorithm and the ability of the prediction. The formulas are as follows:

$$
\begin{gathered}
\text { MSE }=\frac{1}{n} \sum_{i=1}^{n}\left(y_{i}^{\prime}-y_{i}\right)^{2}, \\
R \text {-square }=1-\frac{\sum_{i=1}^{n}\left(y_{i}^{\prime}-y_{i}\right)^{2}}{\sum_{i=1}^{n}\left(y_{i}-y_{\text {avg }}\right)^{2}} .
\end{gathered}
$$

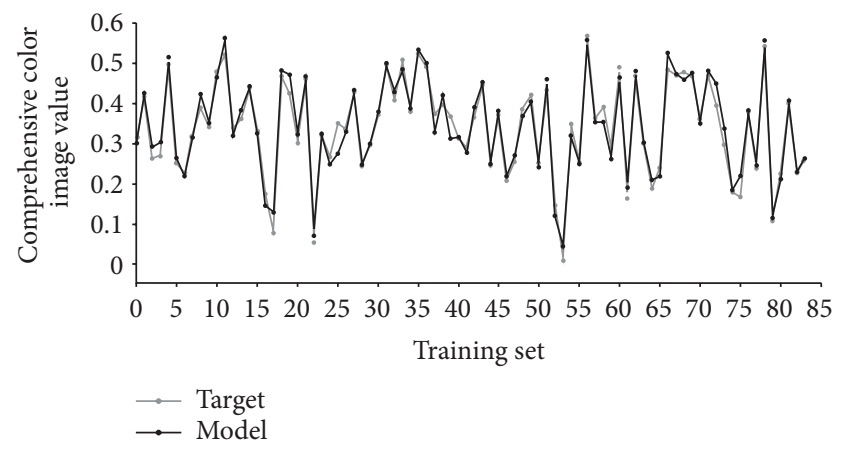

FIgURE 9: The fitting curve of training set.

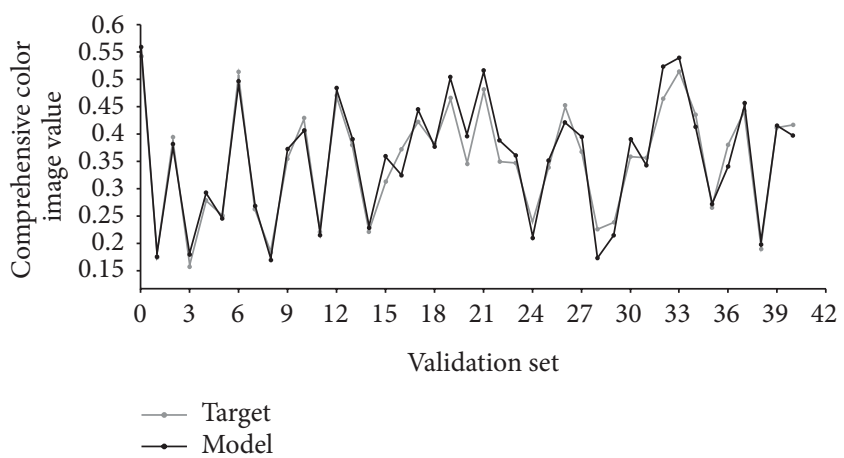

Figure 10: The fitting curve of validation set.

In (13), $y_{i}^{\prime}, y_{i}$, and $y_{\text {avg }}$ represent the predicted value, the actual value, and the actual average value, respectively. The range of $R$-square is $[0,1]$, the closer to 1 , showing that the equation's 6 variables have stronger ability to explain the comprehensive color image $F(x)$. The calculation result shows that the MSE and $R$-square of training set are 0.0006 and 0.9614 , respectively. In order to verify the validity of the GEP model, 41 sets of data are taken into the model, and the curve fitting of validation set is shown in Figure 10. The MSE and $R$-square are 0.0007 and 0.9453 , respectively, achieving the ideal effect.

4.4. Evaluation System. According to the above-mentioned method, VB is used to develop a color image evaluation system for small space. The system can assist designers in color design. In the system, designers input color value and users' factor score of color scheme. According to the function 
TABLE 6: The comparison of evaluation results.

\begin{tabular}{lcccc}
\hline Color scheme & Color value $(\mathrm{R}, \mathrm{G}$, and $\mathrm{B})$ & The value of questionnaire & The value of this method & Deviation \\
\hline 1 & $(112,171,244)$ & 0.7 & 0.6824 & 0.5073 \\
2 & $(229,178,232)$ & 0.55 & 0.1941 & 0.0427 \\
3 & $(250,10,72)$ & 0.2 & 0.5447 & 0.0059 \\
4 & $(32,32,242)$ & 0.55 & 0.4635 & 0.0053 \\
5 & $(123,131,130)$ & 0.45 & 0.5641 & 0.0135 \\
6 & $(159,248,164)$ & 0.55 & 0.2703 & 0.0141 \\
7 & $(240,249,88)$ & 0.25 & 0.2978 & 0.0203 \\
8 & $(112,171,244)$ & 0.3 & 0.7022 & 0.0022 \\
9 & $(235,235,235)$ & 0.75 & 0.7705 \\
\hline
\end{tabular}

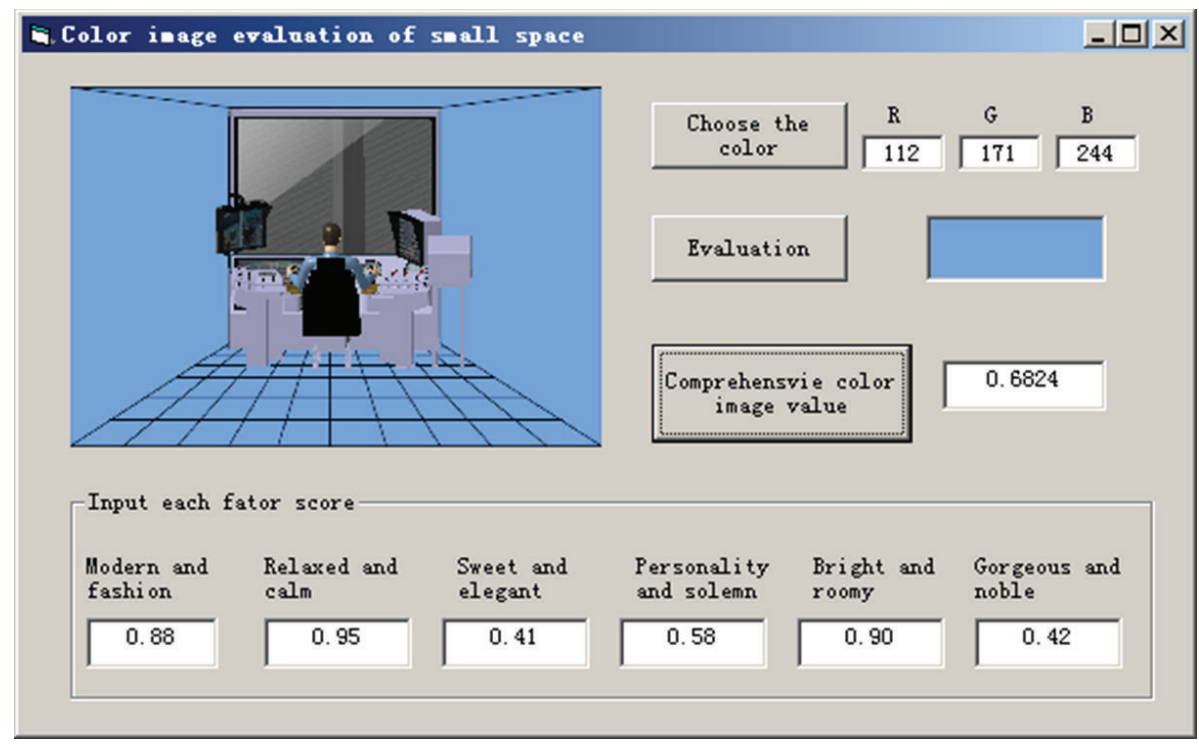

FIGURE 11: The evaluation interface of color image for small space.

constructed by GEP algorithm, the system automatically predicts and outputs comprehensive color image value. As shown in Figure 11, the designer selects color value in the window $(112,171$, and 244), inputs the user rating of the 6 factors: $0.88,0.95,0.41,0.58,0.90$, and 0.42 , and then click on "evaluation" button; the comprehensive color image value of this small space can be calculated, that is, 0.6824 .

In order to verify the color image evaluation methods of small space, 10 unspecified color schemes of small space evaluated by system were evaluated by questionnaire again. 50 subjects (half male and half female) are invited to rate the color scheme; scoring criteria is 0 to 1 . The questionnaire results and the evaluation results using the method proposed in this paper are compared. Table 6 shows that the deviation between the value of questionnaire and above-mentioned method is among 0.0022 0.0478 and the average deviation of 10 color schemes is 0.019 . Therefore, the color image evaluation method for small space put forward in this paper can accurately predict the users' image.

\section{Conclusion}

Image perception is fuzzy, so the color image of small space is uncertain for users. The relationship between all color factors is undefined, and there is complex nonlinear relationship between the comprehensive color image value and color image factors. For the first time, this paper is proposed to apply GEP algorithm to the color design of small space. Based on the research method and framework proposed in this paper, FA was used to preprocess the experimental data, on the premise of reserving the main information data, the correlation of data was removed, and the data dimension was reduced effectively. The training samples were not reduced; moreover, the correlation of the GEP's input variable was eliminated. Combining the entropy weight method to obtain the objective weights of color factors, 125 groups of evaluation data were the data base of prediction model. The color image prediction model for small space realized automatic modeling of complex function based on GEP algorithm. 
The calculation and analysis results of application example showed that the prediction result was close to the target and it had high prediction accuracy. The example of color image evaluation of driller control room proved that GEP had strong nonlinear and global search ability to find function and provided a solution way for rapid evaluation of color image. In the next step of research work, different algorithms will be used to compare the results and analysis. At the same time, the color collocation and the coupling relationship between color and shape will be the research content in next step.

\section{Conflict of Interests}

The authors declare that there is no conflict of interests regarding the publication of this paper.

\section{Acknowledgment}

This research was supported by the National Natural Science Fund Project of China, no. 51105310, and partly supported by the 111 Project, Grant no. B13044.

\section{References}

[1] X.-Y. Wang, Z.-F. Chen, and J.-J. Yun, "An effective method for color image retrieval based on texture," Computer Standards \& Interfaces, vol. 34, no. 1, pp. 31-35, 2012.

[2] X. Y. Wang and Z. Y. Wang, "A novel method for image retrieval based on structure elements' descriptor," Journal of Visual Communication and Image Representation, vol. 24, no. 1, pp. 63-74, 2013.

[3] X. Y. Wang, D. D. Zhang, and X. Guo, "Authentication and recovery of images using standard deviation," Journal of Electronic Imaging, vol. 22, no. 3, Article ID 033012, 2013.

[4] X. Y. Wang, D. D. Zhang, and X. Guo, "A novel image recovery method based on discrete cosine transform and matched blocks," Nonlinear Dynamics, vol. 73, no. 3, pp. 1945-1954, 2013.

[5] S. E. Palmer and K. B. Schloss, "An ecological valence theory of human color preference," Proceedings of the National Academy of Sciences of the United States of America, vol. 107, no. 19, pp. 8877-8882, 2010.

[6] S.-J. Luo, S.-S. Zhu, F.-T. Ying, and J.-S. Zhang, "Statues and progress of research on users' tacit knowledge in product design," Computer Integrated Manufacturing Systems, vol. 16, no. 4, pp. 673-688, 2010.

[7] S.-W. Hsiao, "Fuzzy set theory on car-color design," Color Research \& Application, vol. 19, no. 3, pp. 202-213, 1994.

[8] S. J. Cai, A Study on Building the Color Combination System Using Neural Network and Genetic Algorithm, National Cheng Kung University, Taiwan, China, 2003.

[9] S.-W. Hsiao, C.-F. Hsu, and K.-W. Tang, "A consultation and simulation system for product color planning based on interactive genetic algorithms," Color Research \& Application, vol. 38, no. 5, pp. 375-390, 2013.

[10] Y. Sun, W.-L. Wang, Y.-W. Zhao, and X.-J. Liu, "User image oriented interactive genetic algorithm evaluation mode in product development," Computer Integrated Manufacturing Systems, vol. 18, no. 2, pp. 276-281, 2012.
[11] M. Nagamachi, "Kansei engineering as a powerful consumeroriented technology for product development," Applied Ergonomics, vol. 33, no. 3, pp. 289-294, 2002.

[12] M. Nagamachi, "Kansei engineering: a new ergonomic consumer-oriented technology for product development," International Journal of Industrial Ergonomics, vol. 15, no. 1, pp. 3-11, 1995.

[13] S.-W. Hsiao, F.-Y. Chiu, and C. S. Chen, "Applying aesthetics measurement to product design," International Journal of Industrial Ergonomics, vol. 38, no. 11-12, pp. 910-920, 2008.

[14] S.-W. Hsiao and H.-C. Tsai, "Use of gray system theory in product-color planning," Color Research \& Application, vol. 29, no. 3, pp. 222-231, 2004.

[15] S.-W. Hsiao, F.-Y. Chiu, and H.-Y. Hsu, "A computer-assisted colour selection system based on aesthetic measure for colour harmony and fuzzy logic theory," Color Research \& Application, vol. 33, no. 5, pp. 411-423, 2008.

[16] H.-C. Tsai, S.-W. Hsiao, and F.-K. Hung, "An image evaluation approach for parameter-based product form and color design," Computer-Aided Design, vol. 38, no. 2, pp. 157-171, 2006.

[17] H.-C. Tsai and J.-R. Chou, "Automatic design support and image evaluation of two-coloured products using colour association and colour harmony scales and genetic algorithm," ComputerAided Design, vol. 39, no. 9, pp. 818-828, 2007.

[18] M.-Y. Ma, C.-Y. Chen, and F.-G. Wu, "A design decision-making support model for customized product color combination," Computers in Industry, vol. 58, no. 6, pp. 504-518, 2007.

[19] J. Y. Lin, A Study of Spatial Color Image for Living Space: An Investigation of Living Space for Demostic Units in Taipei, Ming Chuan University, Taiwan, China, 2005.

[20] Y. L. Qin, CognItive Psychology and It's Implications, Posts \& Telecom Press, Beijing, China, 2012.

[21] C. Ferreira, "Gene expression programming in problem solving," in Soft Computing and Industry, pp. 635-653, Springer, London, UK, 2002.

[22] C. R. Liu, The Application of Ergonomics, Shanghai People's Fine Arts Publishing House, Shanghai, China, 2nd edition, 2009.

[23] Y. Zhang, Y. Yang, S. J. Luo, and Y. H. Pan, "Mental construction of user perception image in product design," Journal of Mechanical Engineering, vol. 46, no. 2, pp. 178-184, 2010.

[24] C. E. Osgood, The Measurement of Meaning, University of Illinois Press, Urbana, Ill, USA, 1957.

[25] W. Xue, Statistical Analysis and SPSS Application, China Renmin University Press, Beijing, China, 3rd edition, 2011.

[26] J. G. Zhang and P. S. Vijay, Information Entropy-Theory and Application, China WaterPower Press, Beijing, China, 2012.

[27] C. Ferreira, "Gene expression programming: a new adaptive algorithm for solving problems," Complex Systems, vol. 13, no. 2, pp. 87-129, 2001.

[28] J. Zuo, Core Technology Research on Gene Expression Programming, Sichuan University, Chengdu, China, 2004.

[29] X. Li, C. Zhou, P. C. Nelson, and T. M. Tirpak, "Investigation of constant creation techniques in the context of gene expression programming," in Proceedings of the Genetic and Evolutionary Computation Conference, Seattle, Wash, USA, June 2004.

[30] J. N. Su and H. Q. Li, "Method of product form design based on perceptual image," Chinese Journal of Mechanical Engineering, vol. 40, no. 4, pp. 164-167, 2004. 


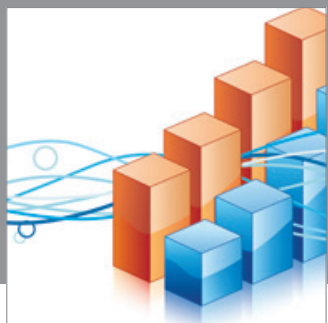

Advances in

Operations Research

mansans

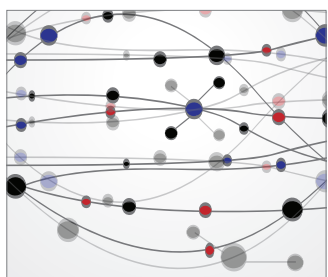

The Scientific World Journal
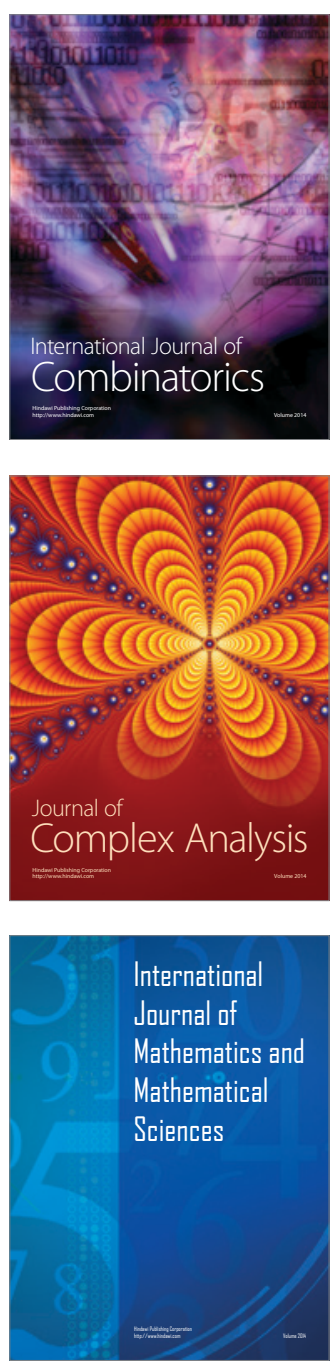
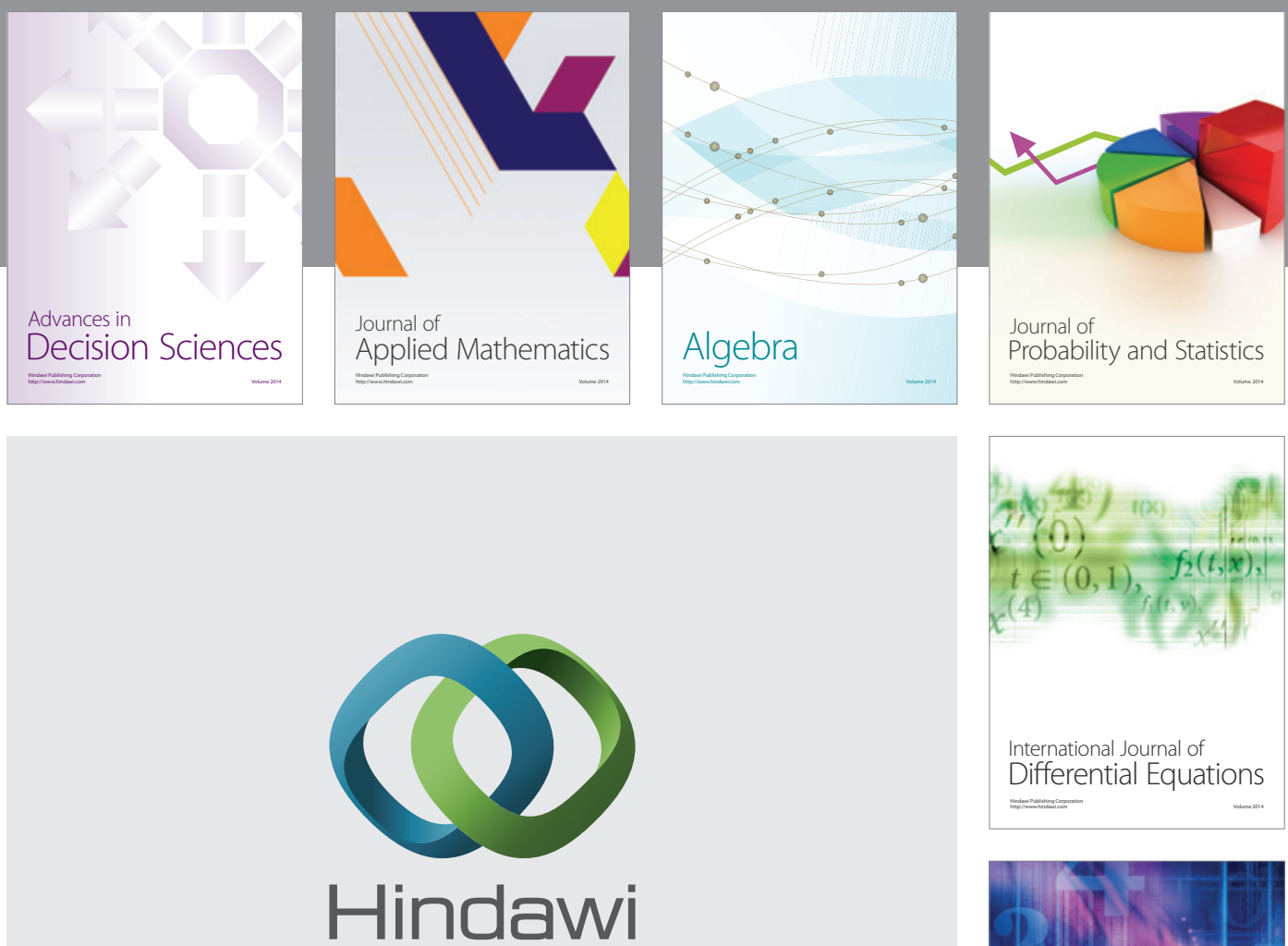

Submit your manuscripts at http://www.hindawi.com
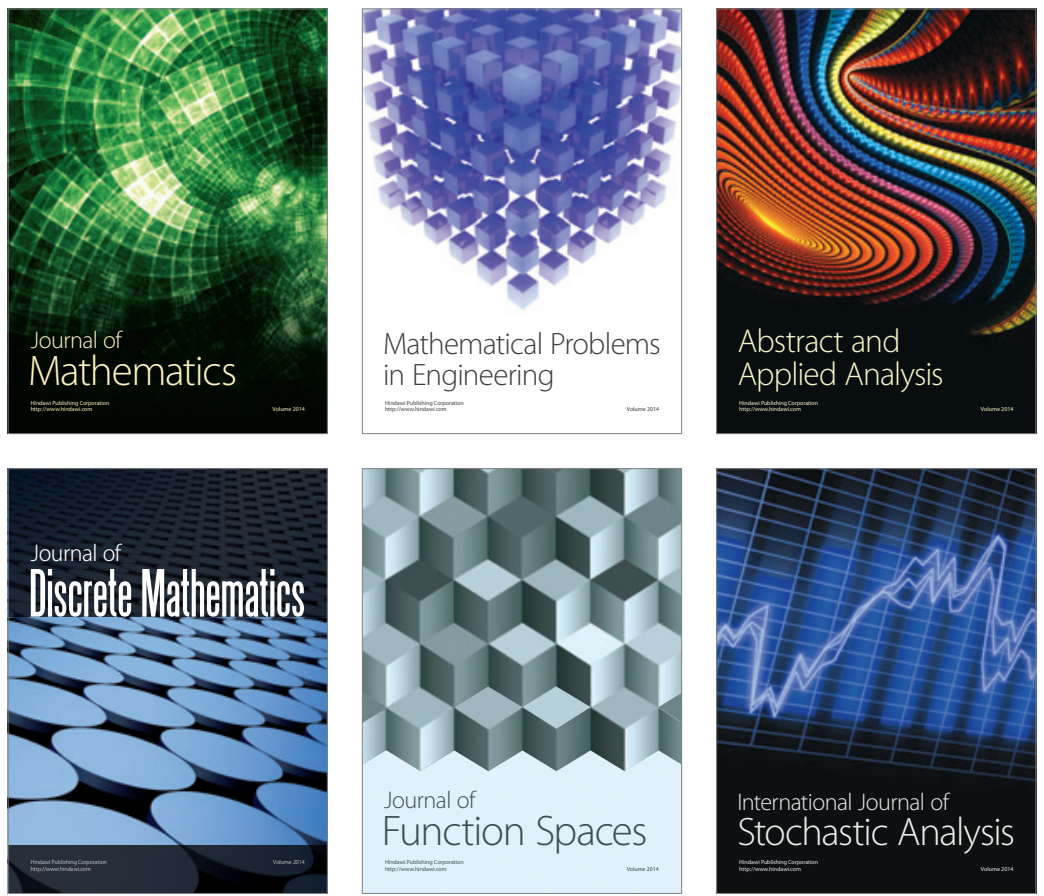

Journal of

Function Spaces

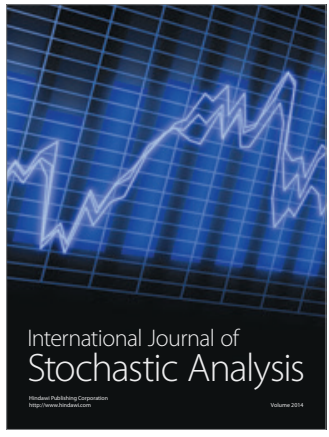

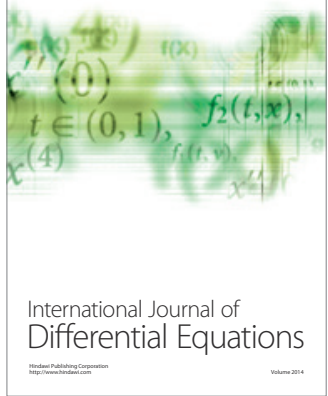
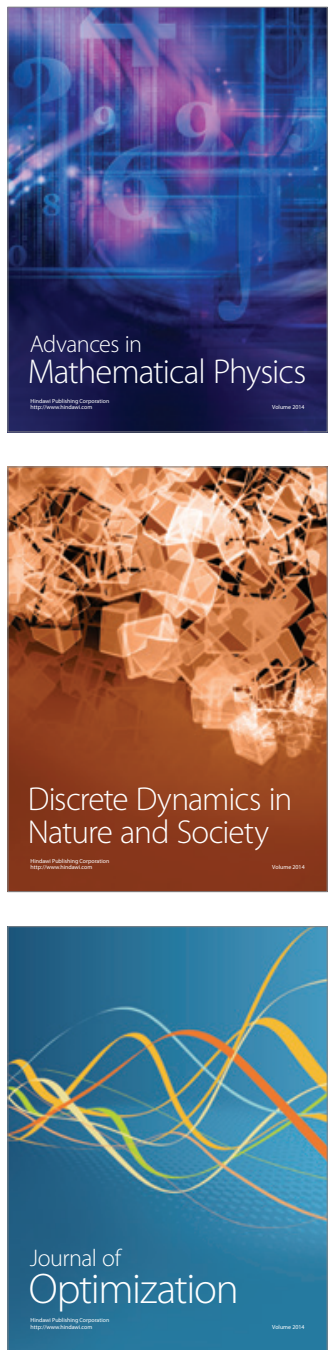